\title{
Changes in Dietary Habits and Eating Behaviors during COVID-19 Induced
}

Confinement

Reem Alnatsheh ${ }^{1}$; MA Alomari ${ }^{1}$, PhD; OF Khabour², Ph.D.; KH Alzoubi ${ }^{3}$, Ph.D.; E Keewan ${ }^{4}, \mathrm{MSc}^{2}$

${ }^{1}$ Department of Physical Education, Qatar University, Doha, Qatar. ${ }^{2}$ Departments of Medical Laboratory Sciences and ${ }^{3} \mathrm{Clinical}$ Pharmacy, Jordan University of Science and Technology, Irbid, Jordan. ${ }^{4}$ Division of Molecular Microbiology, Burnett School of Biomedical Sciences, College of Medicine, University of Central Florida, Orlando, FL 32816, USA

\section{Abstract}

The purpose of this study was to evaluate the dietary habits (DH) and eating behaviors (EB) among adults during COVID19induced confinement. For this purpose, an online survey designed to assess the change in DH and EB during April and May of 2020 was distributed using various social media platforms. A total of 1844 participants aged $18-72$ years completed the survey. The results outlined an increase in most of the $\mathrm{DH}$ and $\mathrm{EB}$ examined in the current study in the majority of the participants. Among these changes, increased prevalence of fruit and vegetable, immune boosters, water, and hot beverage consumption as well as decreased eating in restaurants and fatty food consumption, suggesting a positive change. Conversely, a greater percentage of the participants reported an increase in high calorie food consumption and latenight eating indicating a risky behavior for obesity and subsequent chronic complications. Additionally, age, gender, obesity, education, income, and job type seem to contribute to the changes in DH and EB. Overall, COVID19-induced confinement seems to compel adults to adopt a specific $\mathrm{DH}$ and EB. Though most of these changes were positive, some were negative. The study provides crucial information to design subpopulation recommendations and developmental programs for adults under such conditions.

\section{Introduction}

The coronavirus disease (COVID19) has been declared as a global health pandemic in March 2020.

Transmitted from bats to humans.

Highly contagious infectious disease.

Confinement measures have been implemented to abate the pandemic spread.

These measures impose a severe burden on individual lifestyles, including dietary habits (DH) and eating behaviors (EB).

$\checkmark$ The changes in daily routine due to confinement can potentially make people eat more as a compensatory tactic against fear, boredom, monotony, and anxiety due to uncertainty associated with calamities.

$\checkmark$ On the other hand, some people may turn to practice healthy DH and EB like eating more fresh fruits and vegetables.

$\checkmark$ Given the importance of a well-balanced die for the immune system, possible changes in DH and EB could be a health hazards, especially to during infectious diseases.

However, no studies have examined the effect of COVID19-induced confinement on DH and EB.

\section{Aims of the study}

Determining:

-The changes in DH and EB.

-The factors contributing to these changes.

\section{Methodology}

\section{Participants}

o The study is cross-sectional survey were

distributed during April and May of 2020

Adults (age $>18$ years) of both genders were invited to participate in the study.

- The participants signed an inform consent approved by the IRB.

Survey

Distributed via social media platforms.

Components:

$\checkmark$ Demographics include age, gender, and socioeconomic status (i.e. job, education, and income).

$\checkmark$ Perceptions about COVID19 disease.

$\checkmark$ Changes in DH and EB during the pandemicinduced confinement.

\section{Results}

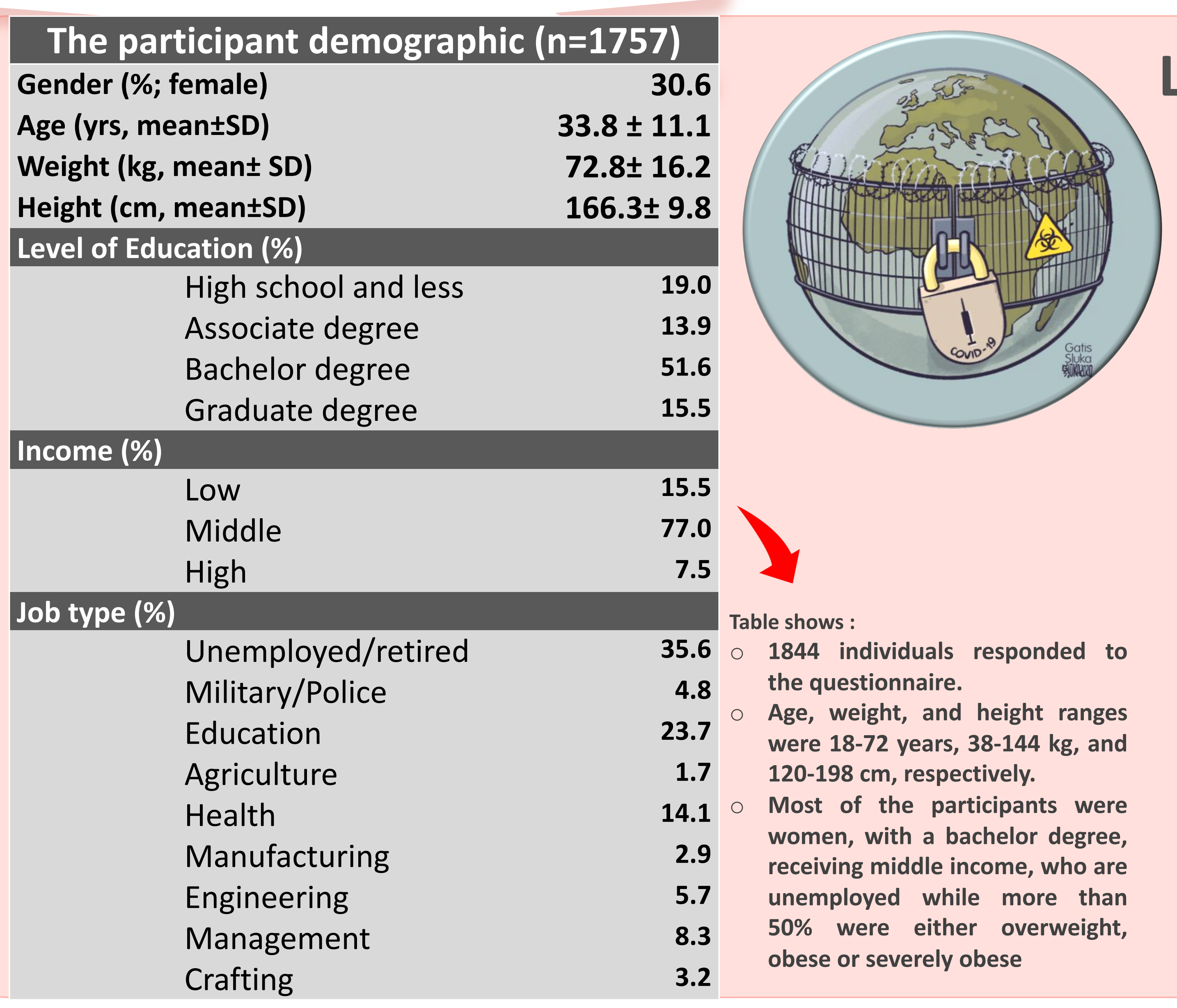

\section{Main Findings}

As previously anticipated (Ref), the current study suggests changes in DH and EB.

$\checkmark$ Most of these changes are in the positive side including consuming an increase in fruit and vegetables, immune boosters, and nutritional supplements and decrease in high-calorie food consumption and late-night eating.

$\checkmark$ Greater percentage reported an increase in eating high-fat foods and dining out.

Age, gender, obesity, education, income, and job type appear to contribute to changes in DH and EB.

$\checkmark$ The type of job contributes to changes in fruit and vegetable consumption

$\checkmark$ Age, gender, education and job type were associated with fat consumption and higher calories.

$\checkmark$ Additionally, age, education, and income were related to supplement consumption.

$\checkmark$ Immune booster consumption was related to obesity, education, income, and job type while water consumption was related to age and education.

$\checkmark$ Consuming hot drinks is linked to age, obesity, and education.

Likelihood of getting infected
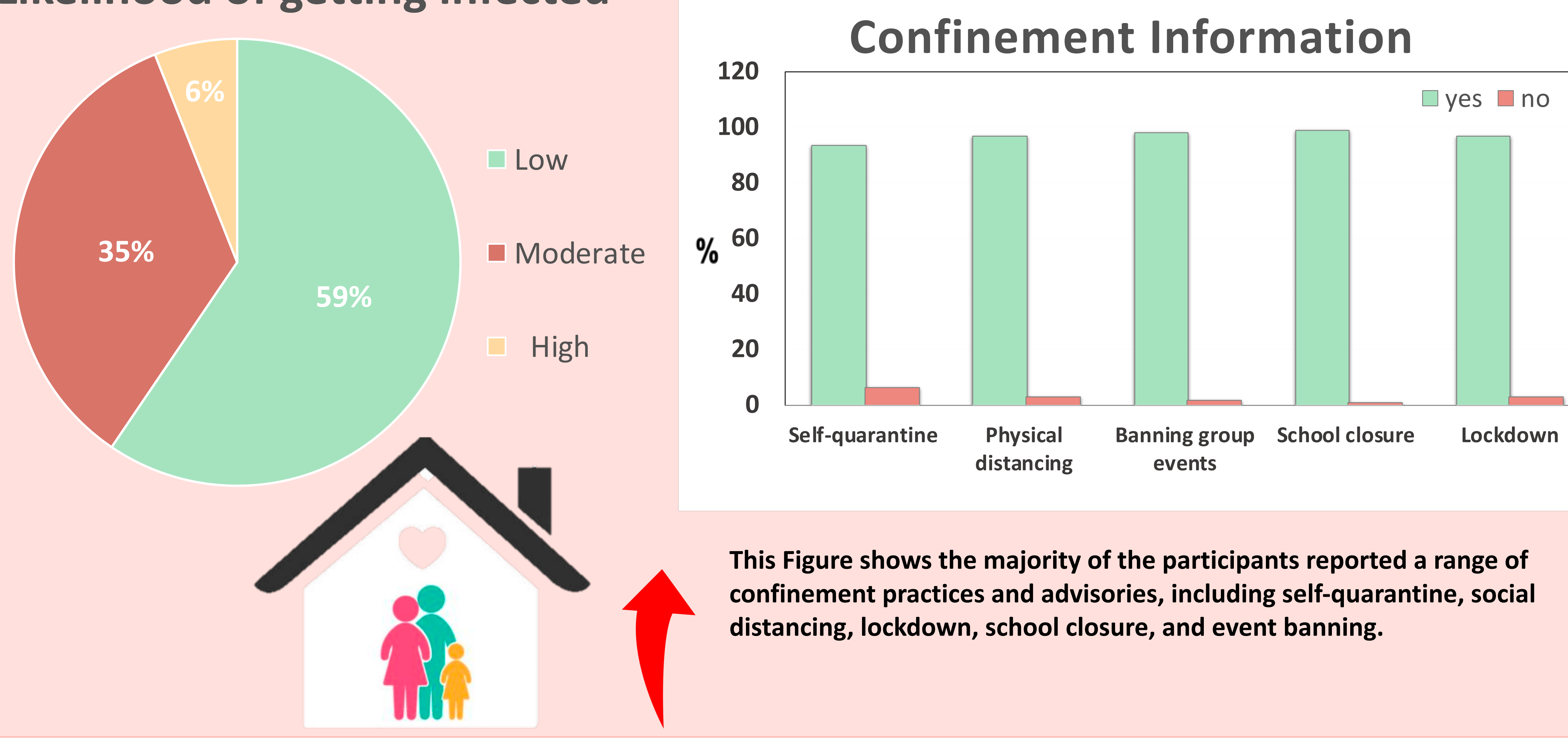

This Figure shows that the majority of the participants reported an increase
$(42.5-61.8 \%)$ in most of the dietary habit measures .
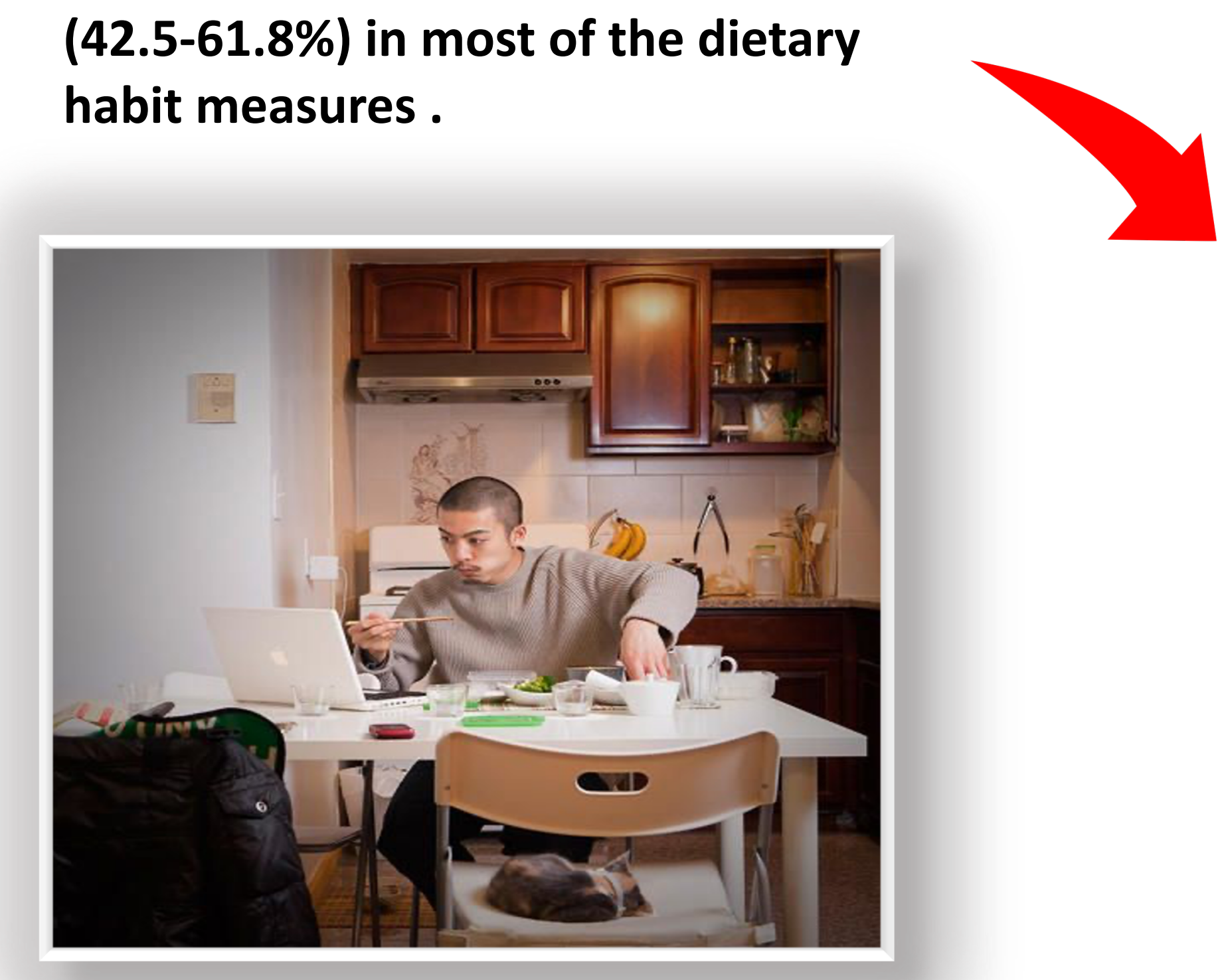

This Figure shows the majority of the participants reported a range of confinement practices and advisories, including self-quarantine, social distancing, lockdown, school closure, and event banning.

\section{Conclusions}

COVID19-induced confinement compels adults to adopt a specific DH and EB in some of the variables examined in the current study.

$\checkmark$ Most of the participants showed improvements in fruit and vegetables, immune boosters, water, hot beverages, nutritional supplements, Eating high-fat foods and dining out.

$\checkmark$ Conversely, a greater percentage of the participants reported risky behaviors including an increase in high calorie food consumption and late-night eating.

Additionally, changes in DH and EB were related to age, gender, obesity, education, income, and job type.

\section{References}

$\checkmark$ Late-night eating was associated with age, and dining at restaurants was associated with age, obesity, and gender. 